\title{
Identification of novel genetic variations affecting osteoarthritis patients
}

\author{
Mamdooh Abdullah Gari ${ }^{1,2,6^{*}}$, Mohammed AlKaff ${ }^{1,4}$, Haneen S. Alsehli, ${ }^{1,2}$, Ashraf Dallol ${ }^{2}$, Abdullah Gari ${ }^{2,3}$, \\ Muhammad Abu-Elmagd ${ }^{5}$, Roaa Kadam ${ }^{5}$, Mohammed F. Abuzinadah²,6, Mazin Gari², Adel M. Abuzenadah²,6, \\ Kalamegam Gauthaman ${ }^{1,5}$, Heba Alkhatabi $i^{5,6}$ and Mohammed M. Abbas ${ }^{1,4}$
}

From 3rd International Genomic Medicine Conference

Jeddah, Saudi Arabia. 30 November - 3 December 2015

\begin{abstract}
Background: Osteoarthritis $(\mathrm{OA})$ is a progressive joint disease characterized by gradual degradation of extracellular matrix (ECM) components in the cartilage and bone. The ECM of cartilage is a highly specified structure that is mainly composed of type II collagen and provides tensile strength to the tissue via aggrecan and proteoglycans. However, changes in the ECM composition and structure can lead to loss of collagen type II and network integrity. Several risk factors have been correlated with OA including age, genetic predisposition, hereditary factors, obesity, mechanical injuries, and joint trauma. Certain genetic association studies have identified several genes associated with OA using genome-wide association studies (GWASs).
\end{abstract}

Results: We identified several novel genetic variants affecting genes that function in several candidate causative pathways including immune responses, inflammatory and cartilage degradation such as SELP, SPN, and COL6A6.

Conclusions: The approach of whole-exome sequencing can be a promising method to identify genetic mutations that can influence the OA disease.

Keywords: Osteoarthritis, Whole-exome sequencing, Single nucleotide variants

\section{Background}

Osteoarthritis $(\mathrm{OA})$ of the knee, which is characterized by gradual degradation of articular cartilage and subchondral bone, is the most common joint disease occurring worldwide [1, 2]. Approximately 27 million individuals in the USA alone are estimated to be diagnosed with OA which affects about $10 \%$ of adults over age 55 years old $[2,3]$. Various risk factors have been associated with OA including genetic predisposition, obesity, gender, mechanical injuries, physical workload, and joint trauma. However, age is the most common factor and affects especially the weight bearing joints, which is often under mechanical stress [4]. The progression of

\footnotetext{
*Correspondence: mgari@kau.edu.sa

'Sheikh Salem Bin Mahfouz Scientific Chair for Treatment of Osteoarthritis by Stem Cells, King Abdulaziz University, Jeddah, Kingdom of Saudi Arabia ${ }^{2}$ Center of Innovation in Personalized Medicine, King Abdulaziz University, Jeddah, Kingdom of Saudi Arabia

Full list of author information is available at the end of the article
}

OA is a consequence of mechanical, biological, biochemical, and molecular factor interactions. What correlation? This correlation can disturb the normal series of synthesis and degradation of articular cartilage chondrocytes and extracellular matrix (ECM) components, and subchondral bone $[5,6]$. In normal cartilage ECM there is a balance between synthesis and degradation that sustain dynamic equilibrium which can be disrupted if microenvironment changes in the ECM composition and structure result in type II collagen loss [7].

Inflammatory stimuli are associated with cartilage ECM changes in OA and play an essential role in the pathogenesis of synovium inflammation and cartilage degeneration through inducing the catabolic activities of chondrocytes $[8,9]$. These Inflammatory factors and cytokines include interleukins family (IL) (IL-1, IL-4, IL-6, IL-17, IL-18), and tumor necrosis factor- $\alpha$ (TNF- $\alpha$ ) [10-14]. For instance, the inflammatory cytokine IL-1 $\beta$ 
is highly expressed in OA stimulating the expression of matrix degradation enzymes including MMP-1, MMP-3, and MMP-13 in chondrocytes and inhibiting ECM synthesis by decreasing the expression SOX9 $[15,16]$. This response ultimately decreases the expression of collagen type II and aggrecan in articular cartilage that lead to reducing the production of chondrogenic ECM [7].

The genetic predisposition to OA is not fully understood. A number of family studies have investigated the genetic nature of OA. Familial mutations were identified in the type II collagen gene (COL2A1) [17]. Mutations affecting collagen IX gene, and COL9A1 have been linked to OA of the hip [18]. Recently, various genetic association studies have identified several genes associated with OA using genome-wide association studies (GWASs). These genes include GDF5, DUS4L, Ch7q22, MCF2L, and GNL3 [19-22]. However, the genetic association between single nucleotide polymorphisms and OA disease remain controversial.

In this study, a pilot whole-exome sequencing analysis was performed on osteoarthritis cases in order to identify candidate gene mutations associated with OA in Saudi Arabia.

\section{Methods}

\section{Patients}

Peripheral blood was obtained from five end-stage osteoarthritis patients with age range of 46-70 years old. The collection of these samples were approved by the King Abdulaziz University Hospital's ethical committee (No.11557). Informed consent was collected from all donors prior to participation in this study.

\section{Whole- exome sequencing}

Genomic DNA was extracted from peripheral blood samples of five OA cases using QIAamp DNA blood mini kit according to the manufacture procedure (Qiagen Inc, USA). Briefly, three micrograms of genomic DNA were fragmented using the Covaris S2 system. Exome capture was performed using the Agilent's SureSelect WholeExome Enrichment kit (v4). Fragment libraries were prepared from the captured exomes for sequencing on the SOLiD 5500 platform (Applied Biosystems). Fragments were sequenced in single reads of $50 \mathrm{bp}$. Reads were aligned to the UCSC hg19 reference sequence of the human genome using the LifeScope analysis pipeline which was also utilized for variants identification and annotation.

\section{PCR amplification and sanger sequencing}

DNA was amplified using custom oligonucleotides primers listed in Table 1. Polymerase chain reaction reactions (PCR) was performed to amplify the selected primers. The PCR reactions were conducted using Go Taq Green Master Mix (Promega, USA). The reactions
Table 1 Primers used for validation of the single nucleotide variations identified by whole-exome sequencing

\begin{tabular}{lll}
\hline Gene & Forward primer $\left(5^{\prime} \rightarrow 3^{\prime}\right)$ & Reverse primer $\left(5^{\prime} \rightarrow 3^{\prime}\right)$ \\
\hline SELP & CCTGTGTAACACAATGCG & CTACAGCTCCTACTACTG \\
FIGNL1 & ATGGTAGCAATGTCAGCAG & GAAGATCGTATCCTAGTGG \\
SPN & CTCGTGCTGAGCAGAGGC & CATCCTCACTGGCCACCAG \\
USP36 & CTCGTGCTGAGCAGAGGC & CTCACTGGCCACCAGTGG \\
TNRC6B & TCTGGCAGAGAACAGGCTC & CACCTGTATTGTTGTCC \\
HSPG2 & GACTCTGCTATGCCATGTC & CACAGGTGGTCAGCGACAC \\
SUSD5 & AGGTGTCTGCTTGACAGTG & CTAGTACTCCTGATCAGC \\
ITGA8 & GGAAGGCTAATAACGATCAG & GGTTAGTAACGTGTGTTCTC \\
PXN & CATCCAAGAGACTCTCCAC & CCATCTGCTGACCTCTAG \\
NLRP6 & AGCAACTGGAGCTTCGTG & GTGGCGCTCGATGTCGCGC \\
COL2A1 & CTAAGTCCAGAGACTGCG & GGCCTGACAGGTCAGCTG \\
COL6A6 & CATCGGTGCTGCACTCAG & CTGTGGTACAGATGTTGG \\
GAS6 & GGCACGCAGCAGATGCAG & CCCAGGCTGGTAGCTGAG \\
\hline
\end{tabular}

conditions were performed at one denaturation cycle at $95^{\circ} \mathrm{C}$ for $15 \mathrm{~min}$ followed by 35 cycles of denaturation at $95^{\circ} \mathrm{C}$ for $30 \mathrm{~s}$, and annealing at $52^{\circ} \mathrm{C}$ for $30 \mathrm{~s}$ and extension at $72^{\circ} \mathrm{C}$ for $30 \mathrm{~s}$. The final step was done at $72^{\circ}$ $\mathrm{C}$ for $10 \mathrm{~min}$. To perform Sanger sequencing, purification of PCR products using ethanol precipitation were required prior to the cycle sequencing reaction. Purified PCR products were labeled with BigDye Terminator kit v3.1 (ABI Biosystem, USA) for cycle sequencing according to the manufacture procedure. After the second purification for the PCR products, the sequencing analysis was performed on the automated sequencer from Applied Biosystems 3500 DNA Analyzer.

\section{Results}

Whole-exome sequencing of $5 \mathrm{OA}$ patients revealed several novel mutations that are predicted to be damaging to protein function (SIFT). Identified variants were prioritized according to their ontology grouping in ECM and/or inflammation (Table 2). A novel c.381G > T, p.Asn127Lys mutation in the gene selectin P (SELP) has not been reported in dbSNP, 1000 Genomes project or the ExAC project. We have validated this mutation by Sanger sequencing and confirmed its existence in the heterozygous state in one patient (Fig. 1). Another novel mutation was identified in the collagen type VI alpha 6 (COL6A6) gene in another OA patient. This heterozygous c.2263T $>$ C, p.Ile1137Thr change was also validated by Sanger sequencing. Other single nucleotide variants were identified that had no minor allele frequency reported by dbSNP. However, they were also found by the ExAC project albeit at a very low frequency (Fig. 1). These genes were identified that impact cartilages extracellular matrix (ECM) organization and cartilage development 
Table 2 Single nucleotide variants identified in our whole-exome screen of OA patients and validated by Sanger sequencing

\begin{tabular}{|c|c|c|c|c|c|c|}
\hline Gene & Chromosome position & AA change & Nucleotide change & Protein ID & $\mathrm{dbSNP}$ & ExAC frequency \\
\hline HSPG2 & $1,22154535, G, A$ & p.Arg4174Cys & c.4391C > T & ENSP00000363827 & rs199899258 & 0.00005066 \\
\hline SUSD5 & $3,33194727, \mathrm{G}, \mathrm{A}$ & p.Thr466Met & c. $629 \mathrm{C}>\mathrm{T}$ & ENSP00000308727 & rs377664152 & 0.00009122 \\
\hline ITGA8 & $10,15628601, G, A$ & p.Ala785Val & c. $1063 C>T$ & ENSP00000367316 & rs371802080 & 0.0000248 \\
\hline PXN & $12,120660505, T, C$ & p.Tyr181Cys & c. $605 \mathrm{~A}>\mathrm{G}$ & ENSP00000267257 & rs371118243 & 0.0001199 \\
\hline COL2A1 & $12,48371204, G, A$ & p.Arg1058Cys & c. $1487 C>T$ & ENSP00000369889 & rs 148350640 & 0.00002493 \\
\hline COL6A6 & 3,130293232,T,C & p.lle1137Thr & c. $2263 \mathrm{~T}>\mathrm{C}$ & ENSP00000351310 & rs 200274210 & 0.006855 \\
\hline FIGNL1 & $7,50513244, A, G$ & p.Met581Thr & c.674T $>C$ & ENSP00000349356 & rs200453649 & 0.000173 \\
\hline USP36 & $17,76803235, T, C$ & p.Arg631Gly & c. $1123 A>G$ & ENSP00000441214 & rs112843316 & 0.000082 \\
\hline SPN & $16,29676061, C, T$ & p.Arg338Cys & c. $400 \mathrm{C}>\mathrm{T}$ & ENSP00000353238 & rs200681097 & 0.000485 \\
\hline TNRC6B & $22,40661502, \mathrm{G}, \mathrm{T}$ & p.Gly423Val & c. $1833 \mathrm{G}>\mathrm{T}$ & ENSP00000401946 & rs201057205 & 0.0003241 \\
\hline SELP & 1,169586366,G,T & p.Asn127Lys & c.381G $>\mathrm{T}$ & ENSP00000263686 & NA & NA \\
\hline NLRP6 & 11,281256,G,T & p.Ala508Ser & $c .892 \mathrm{G}>\mathrm{T}$ & ENSP00000309767 & rs373174851 & 0.000101 \\
\hline GAS6 & $13,114535300, G, A$ & p.Pro415Leu & c. $721 \mathrm{C}>\mathrm{T}$ & ENSP00000349962 & NA & 0.0000353 \\
\hline
\end{tabular}

including heparan sulfate proteoglycan2 (HSPG2), sushi domain containing 5 (SUSD5), integrin subunit alpha 8 (ITGA8), paxillin (PXN), collagen type II alpha 1 (COL2A1), and fidgetin-like 1 (FIGNL1). Moreover, our results revealed some mutations associated in the inflammatory response and immune response such as pyrin domain containing 6 (NLRP6) and growth arrest specific 6 (GAS6); ubiquitin specific peptidase 36 (UPS36), sialophorin (SPN), and trinucleotide repeat containing 6B (TNRC6B) respectively (Table 2 ).

\section{Discussion}

To identify the predisposition genetic factors and the association of these candidate genes with the disease, we conducted a pilot whole-exome sequencing on $5 \mathrm{OA}$ patients from Saudi Arabia. The result of the exome sequence analysis identified several nucleotide variants affecting candidate genes that could likely contribute in OA susceptibility and involved in crucial pathways including cartilage degradation and development, inflammatory, and immune response [5].

The finding of mutations in these genes functionally interrelated to cartilage ECM organization and cartilage signaling pathway may have functional consequences. One of the identified mutations in this study is the p.Arg4174Cys mutation found in the HSPG2 gene. This gene plays multiple roles in the extracellular matrix organization involved in cartilage development, endochondral bone morphogenesis, and chondroitin sulfate metabolic process. Additionally, it is responsible for glycosaminoglycan (GAG) biosynthetic and catabolic process that maintains the expression and aggregation of mature chondrogenic markers [23]. SUSD5 harbors the p.Thr466Met mutation in one OA patient from our cohort. This gene influences the

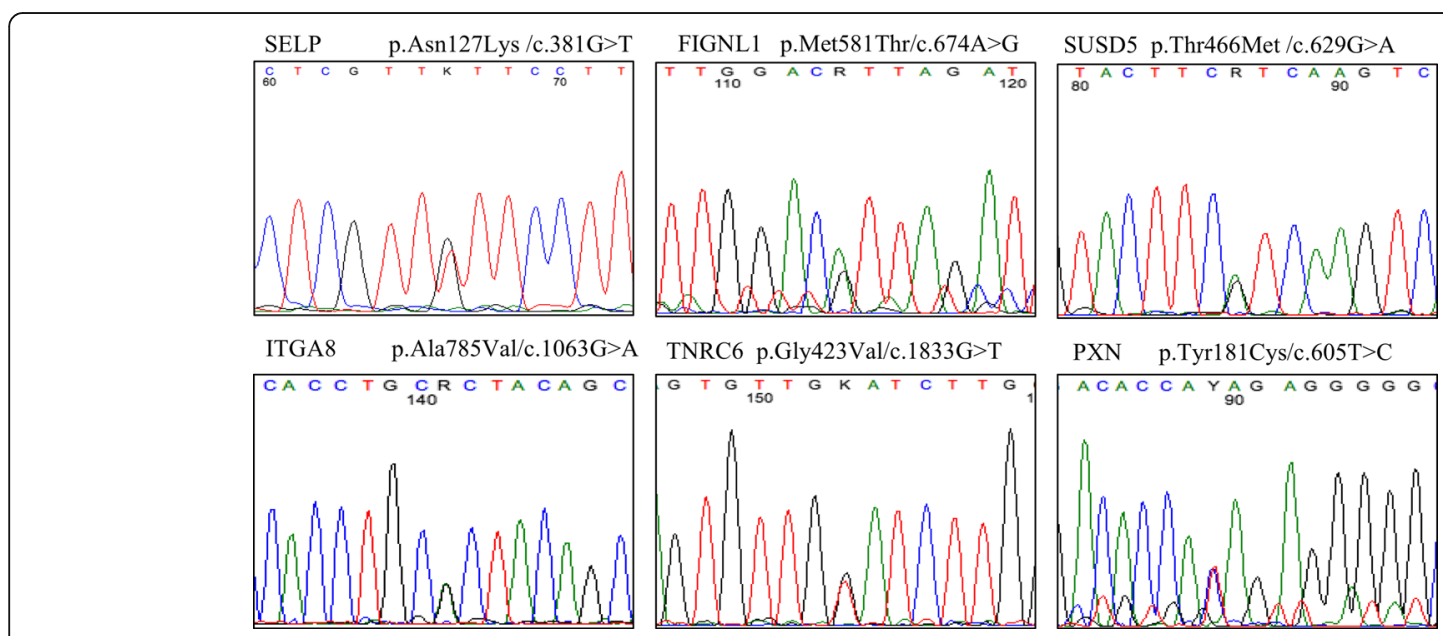

Fig. 1 Selected chromatograms showing the Sanger confirmation of identified variants in our samples 
hyaluronic acid binding, a core component in articular cartilage [24]. Another mutation was identified is the p.Ala785Val mutation in ITGA8, and the p.Tyr181Cys mutation in PXN gene. Both ITGA8 and PXN genes are important in cellular matrix adhesion, ECM organization, and transforming growth factor beta receptor regulation [25, 26]. Most importantly, SNPs variants in cartilage related genes that impacts cartilage development, cartilage condensation, ECM and collagen fibril organization, and collagen catabolic process has been associated in our OA patients. That includes the p.Arg1058Cys mutation in COL2A1, and the p.Ile1137Thr mutation in COL6A6. In addition, the results of this study showed the p.Met581Thr mutation in FIGNL1 gene; a gene that influences the regulatory role in osteoblast proliferation and differentiation [27].

The result of this exome analysis revealed other mutations related to the inflammatory response such as NLRP6 and GAS6; and immune response such as USP36, SPN, and TNRC6B. The identified mutation in UPS36 gene is p.Arg631Gly. This gene is a known member of the ubiquitin specific protease family that regulates the immune signaling pathway [28]. The p.Arg338Cys mutation in SPN was also identified in our patients, and the influence of this gene can affect cellular defense responses and the regulation of the tumor necrosis factor (TNF) pathway [29]. The mutation found in TNRC6B gene is p.Gly423Val; this gene not only act in the innate immune response, but also regulates fibroblast growth factor receptor signaling pathway [30]. Furthermore, the results showed some other mutations involved in the inflammatory response. Interestingly, we identified a novel SNV variation in the SELP, p.Asn127Lys, which has not been reported previously. The function of SELP is to stimulate leukocytes adhesion in the site of injury, which could explain the presence of inflammatory markers IL family, and TNF in OA patients [31]. The p.Ala508Ser mutation in NLRP6 gene, and the p.Pro415Leu mutation in the GAS6 gene were identified in our screen; both genes regulates the inflammatory response [32, 33]. However, GAS6 specifically regulates tumor necrosis factor-mediated signaling pathway and macrophage cytokine production [33].

\section{Conclusions}

OA is a progressive joint disease characterized by gradual degradation of (ECM) components in the cartilage and bone. Several risk factors have been associated with OA such as genetic predisposition and molecular factors. In this study, a pilot whole-exome sequencing was performed and we identified several genes candidate genes associated with $\mathrm{OA}$ in Saudi Arabia that function in various causative pathways including immune response, inflammatory and cartilage degradation. Despite the small sample size of this group, a hypothesis could be formulated that states the importance of personalized genetic screening of OA patients in order to understand the individual's genetic makeup potentially responsible for susceptibility towards OA.

\section{Abbreviations \\ OA: Osteoarthritis; ECM: Extracellular matrix; SNPs: Single nucleotide polymorphisms}

\section{Acknowledgment}

We would like to acknowledge the financial support provided by the Sheikh Salem Bin Mahfouz Scientific Chair for Treatment of Osteoarthritis by Stem Cells. The Center of Innovation in Personalized Medicine (CIPM), Stem Cell Lab at Center of Excellence for Genomic Medicine Research and the Orthopedics Stem Cell Research Lab (CEGMR) at King Abdulaziz University Hospital for supporting this study.

\section{Declaration}

This article has been published as part of BMC Medical Genetics Volume 17 Supplement 1, 2016: Proceedings of the 3rd International Genomic Medicine Conference: medical genetics. The full contents of the supplement are available online at http://bmcmedgenet.biomedcentral.com/articles/ supplements/volume-17-supplement-1.

\section{Funding}

This study was funded by the Sheikh Salem Bin Mahfouz Scientific Chair for Treatment of Osteoarthritis by Stem Cells. Publication fees for this article are paid by the Center of Excellence in Genomic Medicine Research (CEGMR), King Abdulaziz University, Jeddah, Kingdom of Saudi Arabia.

\section{Availability of data and materials \\ Not applicable.}

\section{Authors' contributions}

MAG, AMA, MAE, AD and MMA participated in the study design. MA, MAG, AG, MG and HSA performed data collection, DNA extraction and sequencing studies. KG, HA, RK, MAE and AD analyzed data, interpreted the results and drafted the manuscript. MAG, AMA, MMA, and MFA participated in critical review, editing and finalization of manuscript. All authors read and approved the final manuscript

\section{Competing interests}

The authors declare that they have no competing interests.

\section{Consent for publication}

Not applicable.

\section{Ethics approval and consent to participate}

This study was approved by the Research Committee of the Biomedical Ethics Unit, Faculty of Medicine, King Abdulaziz University, Jeddah, Saudi Arabia. Participants in the study provided their consent prior to sample collection.

\section{Author details}

${ }^{1}$ Sheikh Salem Bin Mahfouz Scientific Chair for Treatment of Osteoarthritis by Stem Cells, King Abdulaziz University, Jeddah, Kingdom of Saudi Arabia. ${ }^{2}$ Center of Innovation in Personalized Medicine, King Abdulaziz University, Jeddah, Kingdom of Saudi Arabia. ${ }^{3}$ Department of Hematology, Faculty of Medicine, King Abdulaziz University Hospital, King Abdulaziz University, Jeddah, Kingdom of Saudi Arabia. ${ }^{4}$ Department of Orthopedic Surgery, Faculty of Medicine, King Abdulaziz University, Jeddah, Kingdom of Saudi Arabia. ${ }^{5}$ Centre of Excellence in Genomic Medicine Research, King Abdulaziz University, Jeddah, Kingdom of Saudi Arabia. ${ }^{6}$ Department of Medical Laboratory Technology, Faculty of Applied Medical Sciences, King Abdulaziz University, P.O. Box 80216, Jeddah 21589, Kingdom of Saudi Arabia.

\section{Published: 10 October 2016}

\section{References}

1. Lane NE, Brandt K, Hawker G, Peeva E, Schreyer E, Tsuji W, Hochberg MC. OARSI-FDA initiative: defining the disease state of osteoarthritis. Osteoarthr Cartil. 2011;19(5):478-82. 
2. Sandell L. Etiology of osteoarthritis: genetics and synovial joint development. Nat Rev Rheumatol. 2012;8(2):77-89.

3. Luo SX, Li S, Zhang XH, Zhang JJ, Long GH, Dong GF, Su W, Deng Y, Liu Y, Zhao JM, et al. Genetic polymorphisms of interleukin-16 and risk of knee osteoarthritis. PLoS One. 2015;10(5), e0123442.

4. Kristjansson B, Honsawek S. Current perspectives in mesenchymal stem cell therapies for osteoarthritis. Stem Cells Int. 2014;2014:194318.

5. Panoutsopoulou K, Zeggini E. Advances in osteoarthritis genetics. J Med Genet. 2013;50(11):715-24

6. van der Kraan PM. Understanding developmental mechanisms in the context of osteoarthritis. Curr Rheumatol Rep. 2013;15(6):333.

7. Maldonado M, Nam J. The role of changes in extracellular matrix of cartilage in the presence of inflammation on the pathology of osteoarthritis. Biomed Res Int. 2013;2013:284873.

8. Brooks P. Inflammation as an important feature of osteoarthritis. Bull World Health Organ. 2003;81(9):689-90.

9. Goldring SR, Goldring MB. The role of cytokines in cartilage matrix degeneration in osteoarthritis. Clin Orthop Relat Res. 2004(427 Suppl):S27-36.

10. Han L, Lee HS, Yoon JH, Choi WS, Park YG, Nam SW, Lee JY, Park WS. Association of IL-17A and IL-17F single nucleotide polymorphisms with susceptibility to osteoarthritis in a Korean population. Gene. 2014;533(1):119-22.

11. Hulin-Curtis SL, Bidwell JL, Perry MJ. Evaluation of IL18 and IL18R1 polymorphisms: genetic susceptibility to knee osteoarthritis. Int J Immunogenet. 2012;39(2):106-9.

12. Kaarvatn MH, Jotanovic Z, Mihelic R, Etokebe GE, Mulac-Jericevic B, Tijanic T, Balen S, Sestan B, Dembic Z. Associations of the interleukin-1 gene locus polymorphisms with risk to hip and knee osteoarthritis: gender and subpopulation differences. Scand J Immunol. 2013;77(2):151-61.

13. Kaewkiattiyot S, Honsawek S, Vejchapipat P, Chongsrisawat V, Poovorawan Y. Association of X-prolyl aminopeptidase 1 rs 17095355 polymorphism with biliary atresia in Thai children. Hepatol Res. 2011:41(12):1249-52.

14. Yigit S, Inanir A, Tekcan A, Tural E, Ozturk GT, Kismali G, Karakus N. Significant association of interleukin-4 gene intron 3 VNTR polymorphism with susceptibility to knee osteoarthritis. Gene. 2014;537(1):6-9.

15. Dai L, Zhang $X, H u X$, Zhou C, Ao Y. Silencing of microRNA-101 prevents IL1 beta-induced extracellular matrix degradation in chondrocytes. Arthritis Res Ther. 2012;14(6):R268

16. Tung JT, Arnold CE, Alexander LH, Yuzbasiyan-Gurkan V, Venta PJ, Richardson DW, Caron JP. Evaluation of the influence of prostaglandin E2 on recombinant equine interleukin-1 beta-stimulated matrix metalloproteinases 1, 3, and 13 and tissue inhibitor of matrix metalloproteinase 1 expression in equine chondrocyte cultures. Am J Vet Res. 2002:63(7):987-93.

17. Knowlton RG, Katzenstein PL, Moskowitz RW, Weaver EJ, Malemud CJ, Pathria MN, Jimenez SA, Prockop DJ. Genetic linkage of a polymorphism in the type II procollagen gene (COL2A1) to primary osteoarthritis associated with mild chondrodysplasia. N Engl J Med. 1990;322(8):526-30.

18. Bateman JF. Genetic aspects of osteoarthritis. Semin Arthritis Rheum. 2005;34(6 Suppl 2):15-8.

19. Day-Williams AG, Southam L, Panoutsopoulou K, Rayner NW, Esko T, Estrada K, Helgadottir HT, Hofman A, Ingvarsson T, Jonsson $H$, et al. A variant in MCF2L is associated with osteoarthritis. Am J Hum Genet. 2011;89(3):446-50.

20. Evangelou E, Valdes AM, Kerkhof HJ, Styrkarsdottir U, Zhu Y, Meulenbelt I, Lories RJ, Karassa FB, Tylzanowski P, Bos SD, et al. Meta-analysis of genomewide association studies confirms a susceptibility locus for knee osteoarthritis on chromosome 7q22. Ann Rheum Dis. 2011;70(2):349-55.

21. Kerkhof HJ, Lories RJ, Meulenbelt I, Jonsdottir I, Valdes AM, Arp P, Ingvarsson $\mathrm{T}$, Jhamai $\mathrm{M}$, Jonsson $\mathrm{H}$, Stolk L, et al. A genome-wide association study identifies an osteoarthritis susceptibility locus on chromosome 7q22. Arthritis Rheum. 2010:62(2):499-510.

22. Miyamoto Y, Mabuchi A, Shi D, Kubo T, Takatori Y, Saito S, Fujioka M, Sudo A, Uchida A, Yamamoto S, et al. A functional polymorphism in the $5^{\prime}$ UTR of GDF5 is associated with susceptibility to osteoarthritis. Nat Genet. 2007:39(4):529-33.

23. Brown AJ, Alicknavitch M, D'Souza SS, Daikoku T, Kirn-Safran CB, Marchetti D, Carson DD, Farach-Carson MC. Heparanase expression and activity influences chondrogenic and osteogenic processes during endochondral bone formation. Bone. 2008;43(4):689-99.

24. Vicent S, Luis-Ravelo D, Anton I, Garcia-Tunon I, Borras-Cuesta F, Dotor J, De Las RJ, Lecanda F. A novel lung cancer signature mediates metastatic bone colonization by a dual mechanism. Cancer Res. 2008:68(7):2275-85.
25. Lu M, Munger JS, Steadele M, Busald C, Tellier M, Schnapp LM. Integrin alpha8beta1 mediates adhesion to LAP-TGFbeta1. J Cell Sci. 2002;115(Pt 23):4641-8.

26. Turner CE. Paxillin and focal adhesion signalling. Nat Cell Biol. 2000;2(12):E231-6.

27. Park SJ, Kim SJ, Rhee Y, Byun JH, Kim SH, Kim MH, Lee EJ, Lim SK. Fidgetinlike 1 gene inhibited by basic fibroblast growth factor regulates the proliferation and differentiation of osteoblasts. J Bone Miner Res. 2007;22(6):889-96.

28. Engel E, Viargues P, Mortier M, Taillebourg E, Coute Y, Thevenon D, Fauvarque MO. Identifying USPs regulating immune signals in Drosophila: USP2 deubiquitinates Imd and promotes its degradation by interacting with the proteasome. Cell Commun Signal. 2014;12:41.

29. Fratazzi C, Manjunath N, Arbeit RD, Carini C, Gerken TA, Ardman B, RemoldO'Donnell E, Remold HG. A macrophage invasion mechanism for mycobacteria implicating the extracellular domain of CD43. J Exp Med. 2000;192(2):183.

30. Edwards TL, Michels KA, Hartmann KE, Velez Edwards DR. BET1L and TNRC6B associate with uterine fibroid risk among European Americans. Hum Genet. 2013;132(8):943-53.

31. Timasheva YR, Nasibullin TR, Imaeva EB, Erdman W, Kruzliak P, Tuktarova IA, Nikolaeva IE, Mustafina OE. Polymorphisms of inflammatory markers and risk of essential hypertension in Tatars from Russia. Clin Exp Hypertens (New York, NY: 1993). 2015;37(5):398-403.

32. Loeser RF, Varnum BC, Carlson CS, Goldring MB, Liu ET, Sadiev S, Kute TE, Wallin R. Human chondrocyte expression of growth-arrest-specific gene 6 and the tyrosine kinase receptor axl: potential role in autocrine signaling in cartilage. Arthritis Rheum. 1997:40(8):1455-65.

33. Tschopp J, Martinon F, Burns K. NALPs: a novel protein family involved in inflammation. Nat Rev Mol Cell Biol. 2003:4(2):95-104.

\section{Submit your next manuscript to BioMed Central and we will help you at every step:}

- We accept pre-submission inquiries

- Our selector tool helps you to find the most relevant journal

- We provide round the clock customer support

- Convenient online submission

- Thorough peer review

- Inclusion in PubMed and all major indexing services

- Maximum visibility for your research

Submit your manuscript at www.biomedcentral.com/submit
) Biomed Central 